\title{
THE NON-PROTEIN NITROGENOUS CONSTITUENTS OF THE BLOOD IN CHRONIC VASCULAR NEPHRITIS (ARTERIOSCLEROSIS) AS INFLUENCED BY THE LEVEL OF PROTEIN METABOLISM *
}

otTo folin, Ph.D., W. Denis, Ph.D., and MalColm Seymour, M.D. BOSTON

By virtue of the analytical technic recently introduced by Folin and Denis for the analysis of blood it has become possible to study the retention of nitrogenous waste products with far greater ease and certainty than formerly. Such studies are particularly to be desired in the investigation of nephritis, for urine analyses cannot reveal the stage of accumulation of such products, an accumulation which may vary between almost normal figures and those accompanying uremia. According to Strauss, the high blood-pressure of nephritis is usually or at least frequently accompanied by an excessive accumulation of the nitrogenous waste products, whereas edema is rather associated with a retention of chlorids. For the investigation reported in this paper we have selected a group of patients whose blood-pressure varied between 160 and $225 \mathrm{~mm}$.

'The chief purpose of our investigation was to determine the extent to which it is possible by means of diets to vary, particularly to reduce, the waste nitrogen in the blood of such nephritis and incidentally determine whether there is any relationship between the blood-pressure and the accumulations of nitrogenous waste products in the blood. With regard to the latter point, the details of our results will be described by one of us (Seymour) in a separate paper, though a few figures for the bloodpressure are recorded in Tables 1 to 12 . We have included in the work a series of "kidney efficiency" tests, according to the phenolsulphonephthalein method recently introduced by Rowntree and Geraghty, in order to obtain further comparisons between the values given by that test and the actual accumulation as revealed by blood analyses. While hospital records were kept of the diet of each patient, we omit all such data and give the urinary nitrogen alone as the index of the total protein metabolism. The subjects of these experiments were inmates of an institution maintained on Long Island, Boston Harbor, by the city of Boston for the accommodation of indigent individuals. They were

* From the Laboratories of Biologic Chemistry and Theory and Practice of Harvard Medical School and the Long Island Hospital.

* Submitted for publication Oct. 6, 1913. 
selected mainly on the basis of abnormally high blood-pressures, and were removed from the institute and placed in a special ward in the Long Island Hospital.

With the exception of Charles M., all of these patients were allowed to be out of bed during the day; but in order to control the collection of urine and the diets, they were not permitted to leave their own ward, except to go on an adjoining veranda. The patients were under the constant supervision of a special nurse and orderly, who were placed at our disposal for this purpose by the hospital authorities.

It was originally our intention to divide the time covered by the experiment into three periods: (1) a period in which the patients would be allowed to eat at will the ordinary ward diet; (2) a period of high protein feeding; (3) a period of low protein diet with high calorific intake. The diets proposed for the first and third periods were successfully administered, but during the period of high protein feeding we were, as will be seen on examination of the figures for urinary nitrogen, unable to raise the protein metabolism to a very high level. This failure was due to the nausea and vomiting which occurred in many cases during that part of the experiments.

During the second or "high protein" period beside the administration of a liberal supply of meat and eggs at the three regular meals, two to three egg-nogs daily were given between meals.

During the third or low protein period the diet consisted of appletapioca pudding, boiled or fried rice, boiled potatoes with butter, fruit (oranges, apples and stewed pears) and boiled cabbage. In addition, during this period, each patient took daily 150 to $200 \mathrm{gm}$. of arrowroot starch, which had been rendered soluble with diastase. This soluble starch was served hot, flavored with fruit juice or with a little port wine or whisky.

During the entire experiment smoking was allowed, and coffee was served three times daily.

The crucial point which we set out to determine, namely, whether by low nitrogen diets it is possible to reduce the urea and total non-protein nitrogen of blood to the normal or less is abundantly proven by the tabular records of the preceding twelve nephritic patients. In every case the figures for the retention nitrogen obtained at the end of the third period, the low protein period, are down substantially to the figures obtained from strictly normal persons (from 22 to $28 \mathrm{mg}$. non-protein nitrogen and from 11 to $14 \mathrm{mg}$. urea nitrogen).

The original level of retention was, to be sure, not very high in several of these patients. The patients had been selected chiefly on the basis of the blood-pressure, and high blood-pressure is not necessarily accompanied by excessive retention of nitrogenous waste products. Judging 
TABLE 1.-Result of Test in Case 1.

Daniel B., aged 60, weight $63.5 \mathrm{~kg}$. Diagnosis: Aortic roughening and regurgitation, chronic interstitial nephritis.

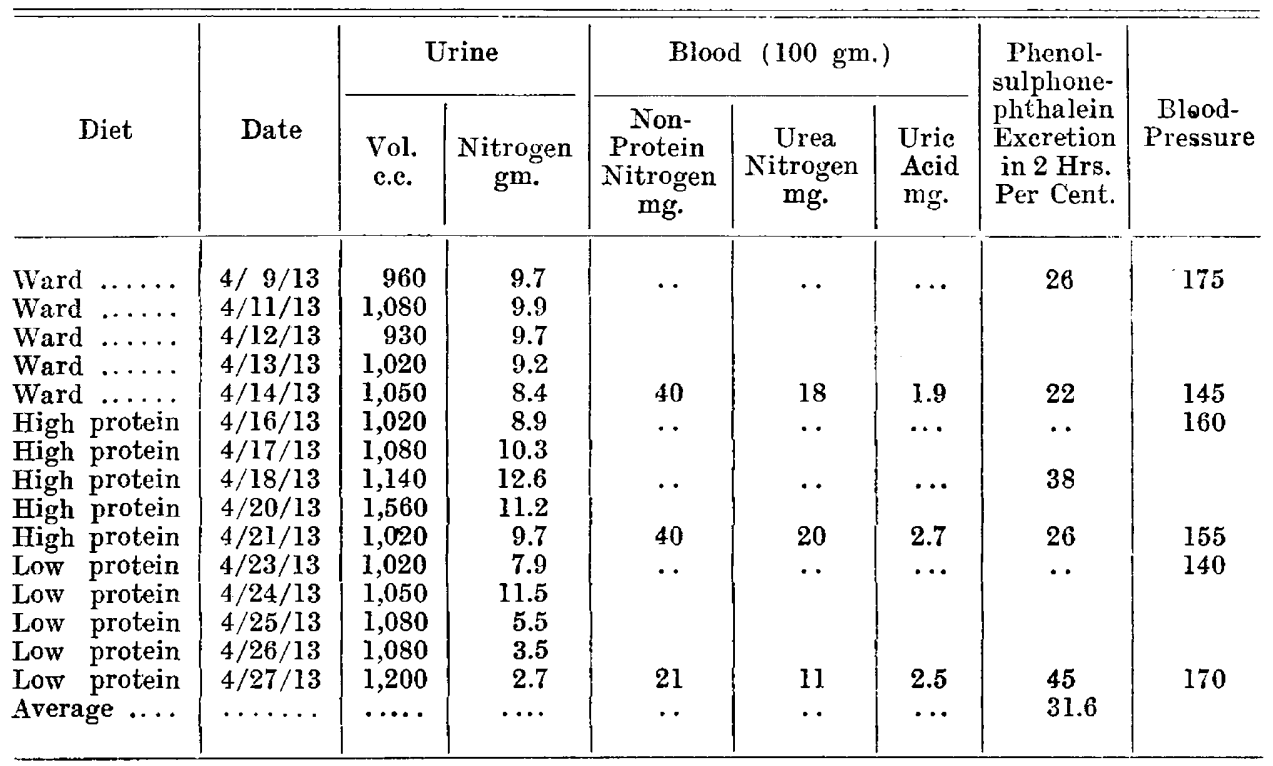

TABLE 2.-Result of Test in Case 2

Thos. H. M., aged 57, weight 80.5 kg. Diagnosis: Chronic interstitial nephritis, hypertrophy of heart, arteriosclerosis.

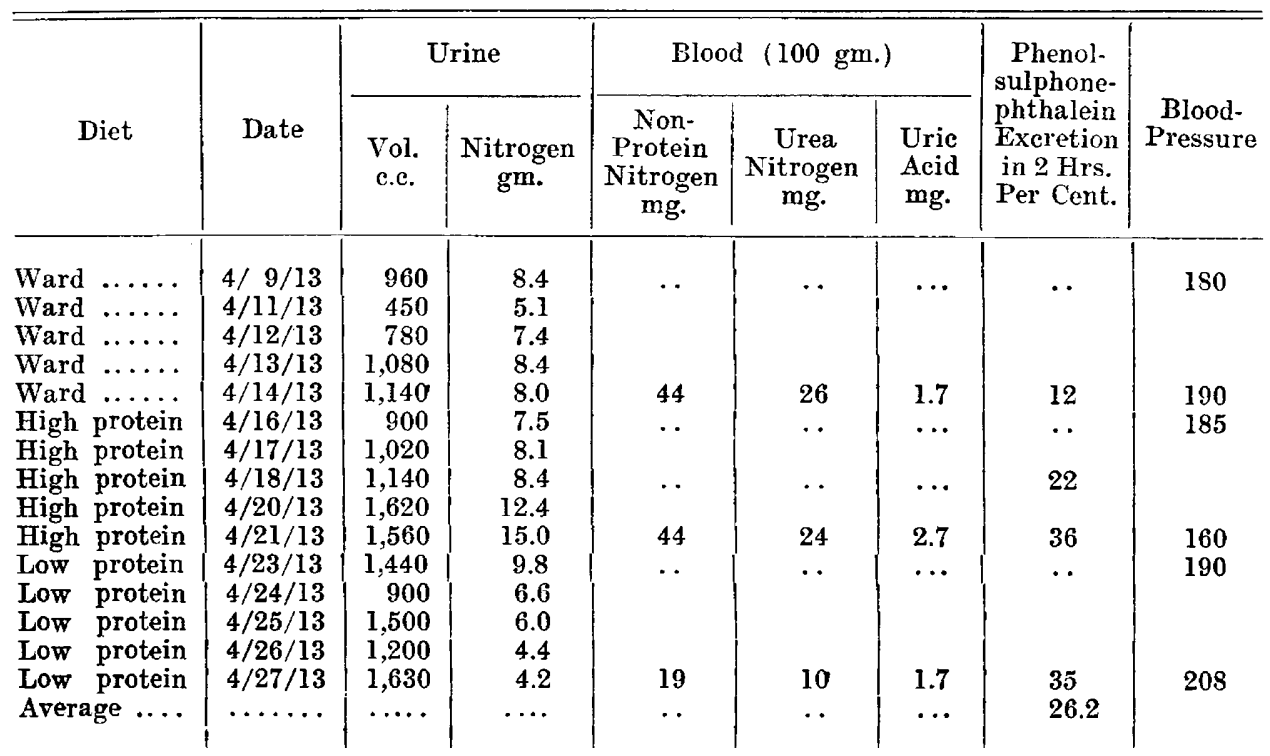


TABLE 3.-Result of Test in CASE 3

William McG., aged 7l, weight $67.5 \mathrm{~kg}$. Diagnosis: Arteriosclerosis, chronic emphysema, chronic interstitial nephritis.

\begin{tabular}{|c|c|c|c|c|c|c|c|c|}
\hline \multirow[b]{2}{*}{ Diet } & \multirow[b]{2}{*}{ Date } & \multicolumn{2}{|c|}{ Urine } & \multicolumn{3}{|c|}{ Blood (100 gm.) } & \multirow{2}{*}{$\begin{array}{l}\text { Phenol- } \\
\text { sulphone- } \\
\text { phthalein } \\
\text { Excretion } \\
\text { in } 2 \text { Hrs. } \\
\text { Per Cent. }\end{array}$} & \multirow[b]{2}{*}{$\begin{array}{l}\text { Blood- } \\
\text { Pressure }\end{array}$} \\
\hline & & $\begin{array}{l}\text { Vol. } \\
\text { c.c. }\end{array}$ & $\begin{array}{l}\text { Nitrogen } \\
\text { gm. }\end{array}$ & $\begin{array}{l}\text { Non- } \\
\text { Protein } \\
\text { Nitrogen } \\
\text { mg. }\end{array}$ & $\begin{array}{c}\text { Urea } \\
\text { Nitrogen } \\
\text { mg. }\end{array}$ & $\begin{array}{l}\text { Uric } \\
\text { Acid } \\
\text { mg. }\end{array}$ & & \\
\hline $\begin{array}{l}\text { Ward } \ldots \ldots \\
\text { Ward } \ldots . . . \\
\text { Ward } \ldots \ldots \\
\text { Ward } \ldots \ldots \\
\text { Ward ...... } \\
\text { High protein } \\
\text { High protein } \\
\text { High protein } \\
\text { High protein } \\
\text { High protein } \\
\text { Low protein } \\
\text { Low protein } \\
\text { Low protein } \\
\text { Low protein } \\
\text { Low protein } \\
\text { Average .... }\end{array}$ & $\begin{array}{l}4 / 9 / 13 \\
4 / 11 / 13 \\
4 / 12 / 13 \\
4 / 13 / 13 \\
4 / 14 / 13 \\
4 / 16 / 13 \\
4 / 17 / 13 \\
4 / 18 / 13 \\
4 / 20 / 13 \\
4 / 21 / 13 \\
4 / 23 / 13 \\
4 / 24 / 13 \\
4 / 25 / 13 \\
4 / 26 / 13 \\
4 / 27 / 13 \\
\ldots \ldots \ldots\end{array}$ & $\begin{array}{r}900 \\
510 \\
900 \\
1,020 \\
1,350 \\
1,020 \\
905 \\
1,020 \\
1,380 \\
1,080 \\
1,920 \\
900 \\
1,440 \\
1,500 \\
1,260 \\
\ldots \ldots\end{array}$ & $\begin{array}{r}7.0 \\
5.1 \\
9.0 \\
7.5 \\
7.8 \\
9.2 \\
8.2 \\
10.8 \\
11.3 \\
10.6 \\
7.4 \\
9.0 \\
5.3 \\
7.1 \\
3.3 \\
\ldots . .\end{array}$ & $\begin{array}{l}\cdots \\
37 \\
\cdots\end{array}$ & $\begin{array}{c}20 \\
\cdots \\
\ldots \\
20 \\
\ldots\end{array}$ & $\begin{array}{l}\cdots \\
2.3 \\
\cdots\end{array}$ & $\begin{array}{c}34 \\
\ldots \\
29 \\
34 \\
\ldots\end{array}$ & $\begin{array}{l}160 \\
145\end{array}$ \\
\hline
\end{tabular}

TABLE 4.-Result of Test in CAse 4

Timothy, W. C., aged 71, weight $91.5 \mathrm{~kg}$. Diagnosis: Right inguinal hernia, arteriosclerosis, senility, chronic interstitial nephritis.

\begin{tabular}{|c|c|c|c|c|c|c|c|c|}
\hline \multirow[b]{2}{*}{ Diet } & \multirow[b]{2}{*}{ Date } & \multicolumn{2}{|c|}{ Urine } & \multicolumn{3}{|c|}{ Blood (100 gm.) } & \multirow{2}{*}{$\begin{array}{l}\text { Phenol- } \\
\text { sulphone- } \\
\text { phthalein } \\
\text { Excretion } \\
\text { in } 2 \text { Hrs. } \\
\text { Per Cent. }\end{array}$} & \multirow[b]{2}{*}{$\begin{array}{l}\text { Blood- } \\
\text { Pressure }\end{array}$} \\
\hline & & $\begin{array}{l}\text { Vol. } \\
\text { c.c. }\end{array}$ & $\begin{array}{l}\text { Nitrogen } \\
\text { gm. }\end{array}$ & $\begin{array}{c}\text { Non- } \\
\text { Nitrogen } \\
\text { Protein } \\
\text { mg. }\end{array}$ & $\begin{array}{c}\text { Urea } \\
\text { Nitrogen } \\
\text { mg. }\end{array}$ & $\begin{array}{l}\text { Uric } \\
\text { Acid } \\
\text { mg. }\end{array}$ & & \\
\hline $\begin{array}{l}\text { Ward } \ldots . . \\
\text { Ward ..... } \\
\text { Ward ...... } \\
\text { Ward ...... } \\
\text { Ward ...... } \\
\text { High protein } \\
\text { High protein } \\
\text { High protein } \\
\text { High protein } \\
\text { High protein } \\
\text { Low protein } \\
\text { Low protein } \\
\text { Low protein } \\
\text { Low protein } \\
\text { Low protein } \\
\text { Average .... }\end{array}$ & $\begin{array}{l}4 / 9 / 13 \\
4 / 11 / 13 \\
4 / 12 / 13 \\
4 / 13 / 13 \\
4 / 14 / 13 \\
4 / 16 / 13 \\
4 / 17 / 13 \\
4 / 18 / 13 \\
4 / 20 / 13 \\
4 / 21 / 13 \\
4 / 23 / 13 \\
4 / 24 / 13 \\
4 / 25 / 13 \\
4 / 26 / 13 \\
4 / 27 / 13 \\
\ldots \ldots \ldots\end{array}$ & $\begin{array}{r}\ldots \\
570 \\
1,560 \\
1,230 \\
2,070 \\
1,560 \\
1,440 \\
1,560 \\
1,080 \\
1,440 \\
1,080 \\
1,050 \\
1,620 \\
1,980 \\
2,040 \\
\ldots \ldots\end{array}$ & $\begin{array}{r}\ldots . . \\
5.4 \\
11.2 \\
8.8 \\
12.0 \\
10.1 \\
13.7 \\
14.8 \\
16.2 \\
18.7 \\
8.7 \\
10.4 \\
6.5 \\
5.9 \\
5.2 \\
\ldots \ldots\end{array}$ & $\begin{array}{l}49 \\
\ldots \\
\ldots \\
48 \\
\ldots\end{array}$ & $\begin{array}{c}25 \\
\cdots \\
\cdots \\
23 \\
\ldots\end{array}$ & $\begin{array}{c}2.6 \\
\cdots \\
\cdots \\
2.6 \\
\ldots\end{array}$ & $\begin{array}{l}\cdots \\
\cdots \\
31 \\
39 \\
\cdots\end{array}$ & $\begin{array}{l}170 \\
142\end{array}$ \\
\hline
\end{tabular}




\section{TABLE 5.-Result of Test in Case 5}

Nathan, D. L., aged 81, weight $78.5 \mathrm{~kg}$. Diagnosis: Arteriosclerosis, senility, cardiac hypertrophy, chronic interstitial nephritis.

\begin{tabular}{|c|c|c|c|c|c|c|c|c|}
\hline \multirow[b]{2}{*}{ Diet } & \multirow[b]{2}{*}{ Date } & \multicolumn{2}{|c|}{ Urine } & \multicolumn{3}{|c|}{ Blood (100 gm.) } & \multirow{2}{*}{$\begin{array}{l}\text { Phenol- } \\
\text { sulphone- } \\
\text { phthalein } \\
\text { Excretion } \\
\text { in } 2 \text { Hrs. } \\
\text { Per Cent. }\end{array}$} & \multirow[b]{2}{*}{$\begin{array}{l}\text { Blood- } \\
\text { Pressure }\end{array}$} \\
\hline & & $\begin{array}{l}\text { Vol. } \\
\text { c.c. }\end{array}$ & $\begin{array}{l}\text { Nitrogen } \\
\text { gm. }\end{array}$ & $\begin{array}{l}\text { Non- } \\
\text { Protein } \\
\text { Nitrogen } \\
\text { mg. }\end{array}$ & $\begin{array}{c}\text { Urea } \\
\text { Nitrogen } \\
\text { mg. }\end{array}$ & $\begin{array}{l}\text { Uric } \\
\text { Acid } \\
\text { mg. }\end{array}$ & & \\
\hline $\begin{array}{l}\text { Ward } \ldots \ldots \\
\text { Ward } \ldots \ldots \\
\text { Ward } \ldots \ldots \\
\text { Ward } \ldots \ldots \\
\text { Ward } \ldots \ldots \\
\text { High protein } \\
\text { High protein } \\
\text { High protein } \\
\text { High protein } \\
\text { High protein } \\
\text { Low protein } \\
\text { Low protein } \\
\text { Low protein } \\
\text { Low protein } \\
\text { Low protein } \\
\text { Average .... }\end{array}$ & $\begin{array}{l}4 / 9 / 13 \\
4 / 11 / 13 \\
4 / 12 / 13 \\
4 / 13 / 13 \\
4 / 14 / 13 \\
4 / 16 / 13 \\
4 / 17 / 13 \\
4 / 18 / 13 \\
4 / 20 / 13 \\
4 / 21 / 13 \\
4 / 23 / 13 \\
4 / 24 / 13 \\
4 / 25 / 13 \\
4 / 26 / 13 \\
4 / 27 / 13 \\
\ldots \ldots \ldots\end{array}$ & $\begin{array}{r}1,290 \\
600 \\
1,110 \\
1,140 \\
1,590 \\
1,260 \\
990 \\
1,200 \\
1,440 \\
1,050 \\
1,440 \\
1,080 \\
960 \\
1,500 \\
1,920 \\
\ldots \ldots\end{array}$ & $\begin{array}{r}7.8 \\
5.7 \\
7.8 \\
8.5 \\
12.2 \\
8.4 \\
8.5 \\
12.0 \\
11.0 \\
10.0 \\
6.7 \\
6.9 \\
4.5 \\
5.7 \\
4.3 \\
\ldots \ldots\end{array}$ & $\begin{array}{c}50 \\
\ldots \\
\ldots \\
58 \\
\ldots\end{array}$ & $\begin{array}{l}28 \\
\ldots \\
\ldots\end{array}$ & $\begin{array}{c}2.7 \\
\ldots \\
\ldots \\
3.3 \\
\ldots\end{array}$ & $\begin{array}{c}14 \\
\ldots \\
20 \\
17 \\
\ldots\end{array}$ & $\begin{array}{l}235 \\
170\end{array}$ \\
\hline
\end{tabular}

TABLE 6.-Result of Test IN CAse 6

Martin, T. D., aged 54, weight $71.5 \mathrm{~kg}$. Diagnosis: Arteriosclerosis, chronic interstitial nephritis.

\begin{tabular}{|c|c|c|c|c|c|c|c|c|}
\hline \multirow[b]{2}{*}{ Diet } & \multirow[b]{2}{*}{ Date } & \multicolumn{2}{|c|}{ Urine } & \multicolumn{3}{|c|}{ Blood (100 gm.) } & \multirow{2}{*}{$\begin{array}{l}\text { Phenol- } \\
\text { sulphone- } \\
\text { phthalein } \\
\text { Excretion } \\
\text { in } 2 \text { Hrs. } \\
\text { Per Cent. }\end{array}$} & \multirow[b]{2}{*}{$\begin{array}{c}\text { Pressure } \\
\text { Blood- }\end{array}$} \\
\hline & & $\begin{array}{l}\text { Vol. } \\
\text { c.c. }\end{array}$ & $\begin{array}{c}\text { Nitrogen } \\
\text { gm. }\end{array}$ & $\begin{array}{c}\text { Non- } \\
\text { Protein } \\
\text { Nitrogen } \\
\text { mg. }\end{array}$ & $\begin{array}{c}\text { Urea } \\
\text { Nitrogen } \\
\text { mg. }\end{array}$ & $\begin{array}{l}\text { Uric } \\
\text { Acid } \\
\text { mg. }\end{array}$ & & \\
\hline Ward ..... & $4 / 9 / 13$ & 1,260 & 11.1 & $\cdots$ & $\cdots$ & . & 35 & 235 \\
\hline Ward .. & $4 / 11 / 13$ & 1,050 & 10.5 & & & & & \\
\hline Ward .. & $4 / 12 / 13$ & 930 & 6.4 & & & & & \\
\hline Ward .. & $4 / 13 / 13$ & 1,530 & 7.8 & & & & & \\
\hline Ward & $4 / 14 / 13$ & 1,710 & 8.7 & 39 & 18 & 3.0 & 33 & 187 \\
\hline High protein & $4 / 16 / 13$ & 1,020 & 8.3 & . & . & $\ldots$ & . & 200 \\
\hline High protein & $4 / 17 / 13$ & 1,500 & 13.1 & & & & & \\
\hline High protein & $4 / 18 / 13$ & 1,260 & 9.6 & . & . & $\ldots$ & 23 & \\
\hline High protein & $4 / 20 / 13$ & 2,040 & 14.1 & & & & & \\
\hline High protein & $4 / 21 / 13$ & 1,620 & 13.6 & 62 & 45 & 3.9 & 30 & 180 \\
\hline Low protein & $4 / 23 / 13$ & 1,320 & 8.5 & . & . & $\cdots$ & . & 160 \\
\hline Low protein & $4 / 24 / 13$ & 1,200 & 7.7 & & & & & \\
\hline Low protein & $4 / 25 / 13$ & 1,500 & 6.1 & & & & & \\
\hline Low protein & $4 / 26 / 13$ & 1,560 & 5.6 & & & & & \\
\hline Low protein & $4 / 27 / 13$ & 1,560 & 4.8 & 30 & 15 & 2.7 & 29 & 190 \\
\hline Average .... & $\ldots \ldots$ & $\cdots$ & $\cdots$ & $\cdots$ & $\cdots$ & $\cdots$ & 30 & \\
\hline
\end{tabular}


TABLE 7.-Result of Test in Case 7

Edward R., aged 74, weight $59.5 \mathrm{~kg}$. Diagnosis: Inguinal hernia, arteriosclerosis, chronic interstitial nephritis.

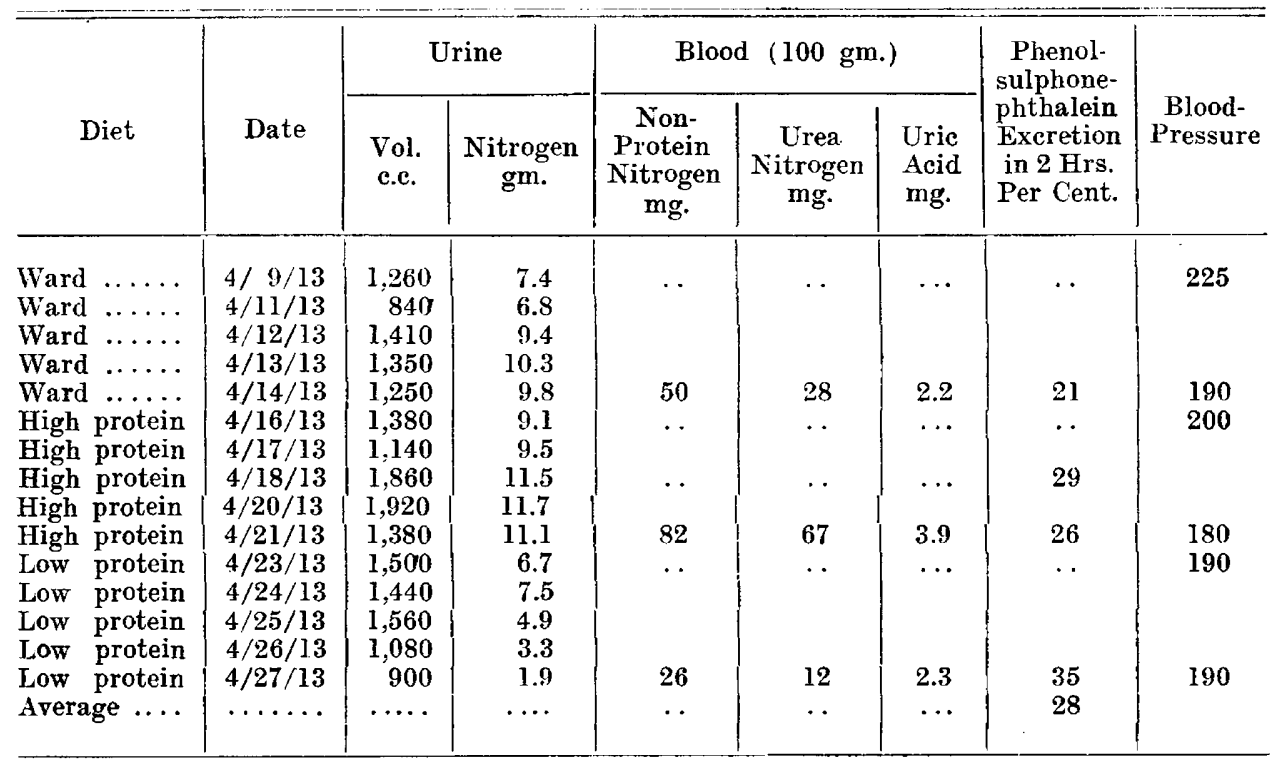

TABLE 8.-Result of Test in CASE 8

Michael $0^{\prime} \mathrm{C}$, aged 66, weight $90.5 \mathrm{~kg}$. Diagnosis: Arteriosclerosis, chronic emphysema, cardiac hypertrophy, chronic interstitial nephritis.

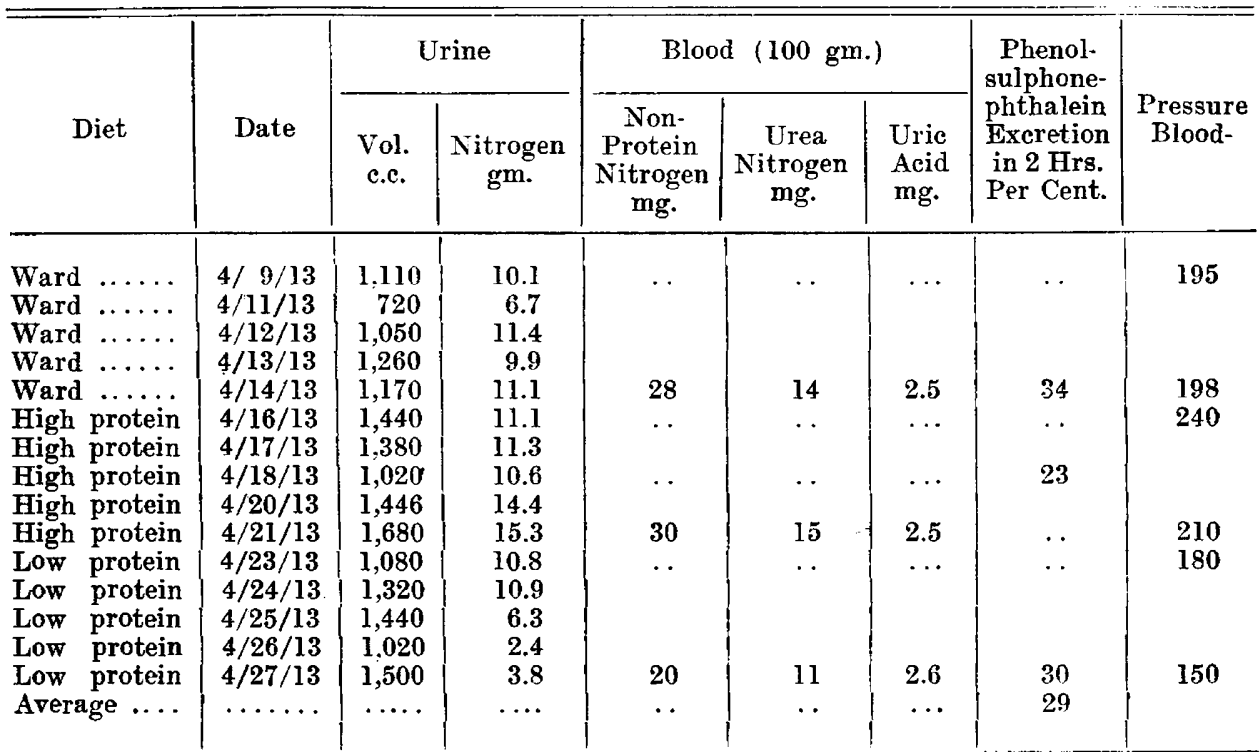


TABLE 9.-Result of Test in Case 9

William T. W., aged 59 , weight $70.5 \mathrm{~kg}$. Diagnosis: Left hemiplegia, mitral regurgitation and aortic roughening, chronic interstitial nephritis, arteriosclerosis.

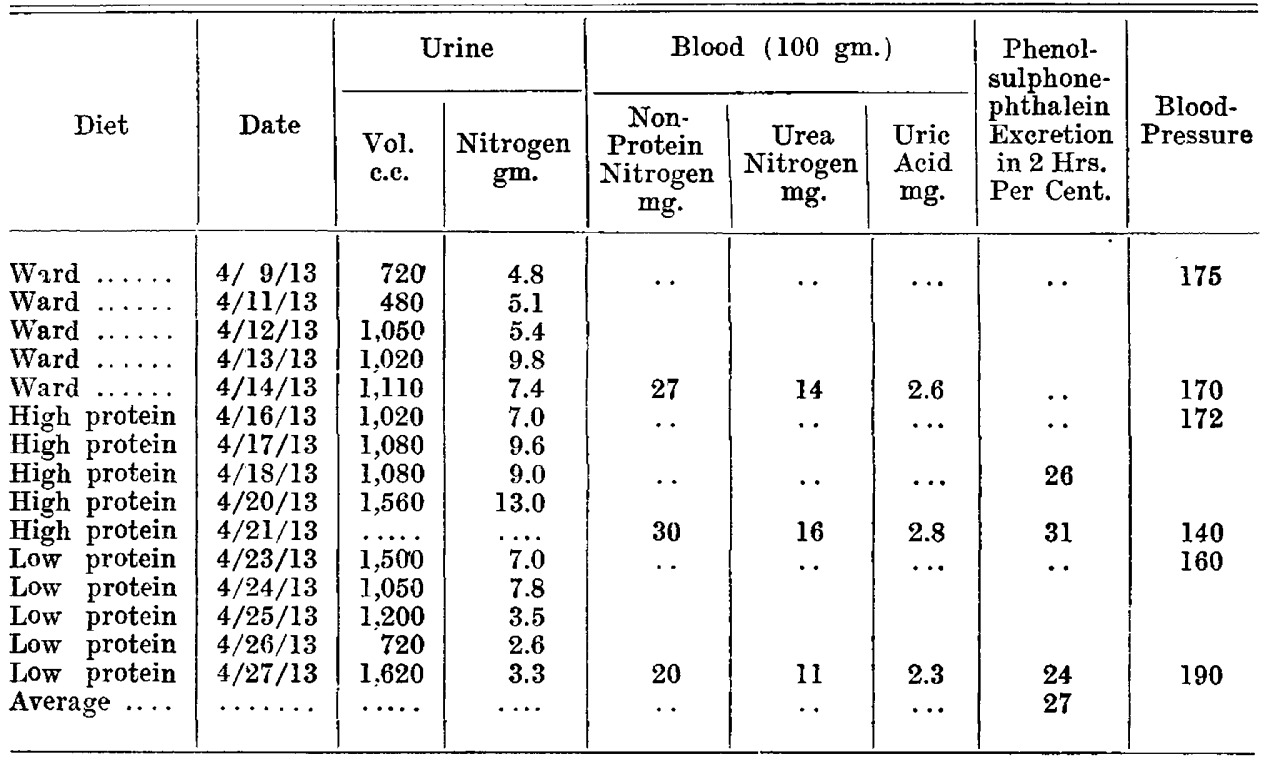

TABLE 10.--Result of Test in Case 10

John DeW., aged 82 , weight $80 \mathrm{~kg}$. Diagnosis: Cardiac hypertrophy, arteriosclerosis, chronic interstitial nephritis.

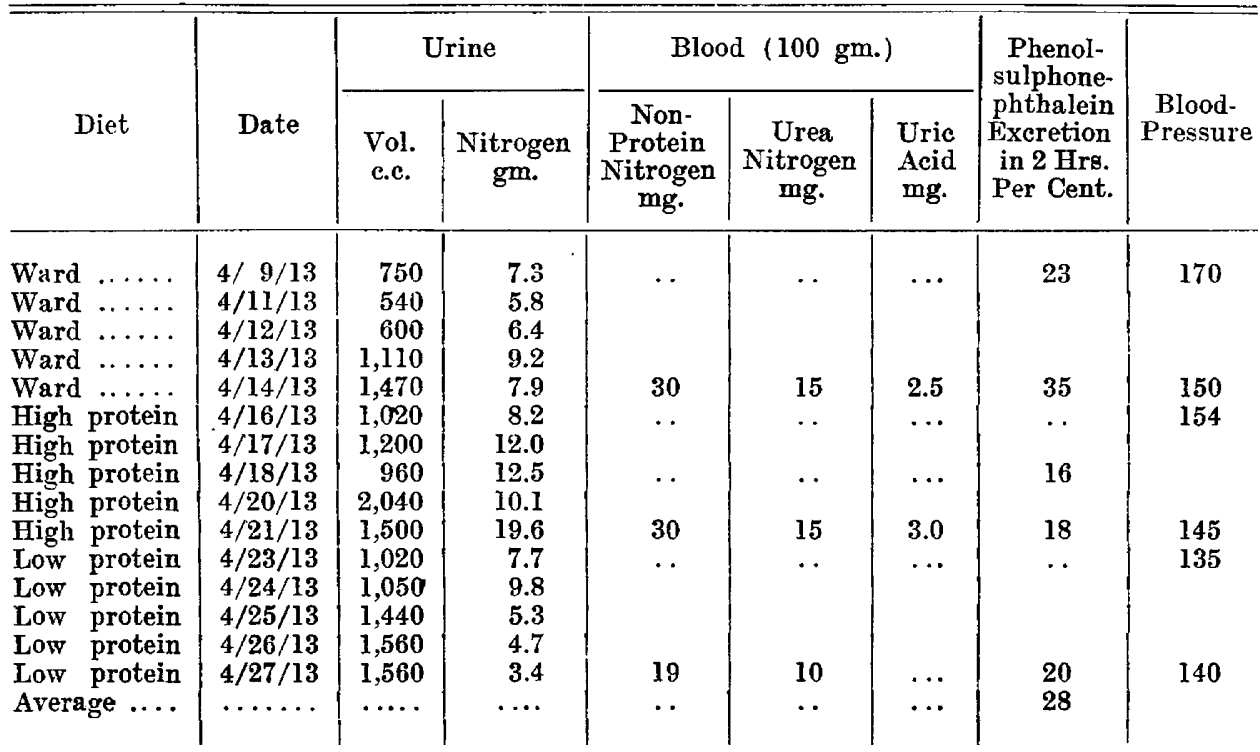




\section{TABLE 11.-Result of Test in CASE 11}

Frank M., aged 72, weight $89.5 \mathrm{~kg}$. Diagnosis: Arteriosclerosis, cardiac hypertrophy, elhronic interstitial nephritis.

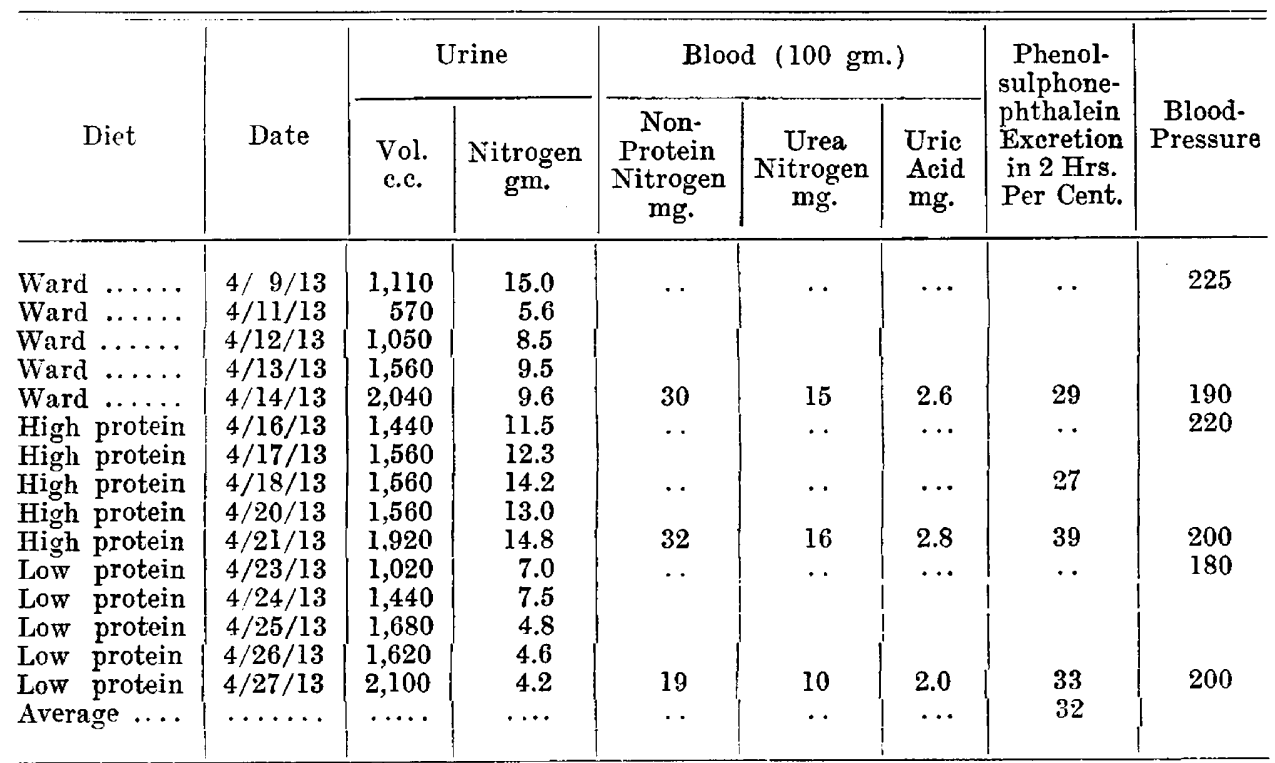

TABLE 12.-Result of Test in Case 12

Chas. McL., aged 63, weight $90.5 \mathrm{~kg}$. Diagnosis: Cardiac hypertrophy, arteriosclerosis, chronic interstitial nephritis.

\begin{tabular}{|c|c|c|c|c|c|c|c|c|}
\hline \multirow[b]{2}{*}{ Diet } & \multirow[b]{2}{*}{ Date } & \multicolumn{2}{|c|}{ Urine } & \multicolumn{3}{|c|}{ Blood (100 gm.) } & \multirow{2}{*}{$\begin{array}{l}\text { Phenol- } \\
\text { sulphone- } \\
\text { phthalein } \\
\text { Excretion } \\
\text { in } 2 \text { Hrs. } \\
\text { Per Cent. }\end{array}$} & \multirow[b]{2}{*}{$\begin{array}{c}\text { Pressure } \\
\text { Blood. }\end{array}$} \\
\hline & & $\begin{array}{l}\text { c.c. } \\
\text { Vol. }\end{array}$ & $\begin{array}{l}\text { Nitrogen } \\
\text { gm. }\end{array}$ & $\begin{array}{c}\text { Non- } \\
\text { Protein } \\
\text { Nitrogen } \\
\text { mg. }\end{array}$ & $\begin{array}{l}\text { Urea } \\
\text { Nitrogen } \\
\text { mg. }\end{array}$ & $\begin{array}{l}\text { Uric } \\
\text { Acid } \\
\text { mg. }\end{array}$ & & \\
\hline $\begin{array}{l}\text { Ward } \ldots \ldots \\
\text { Ward ..... } \\
\text { Ward ...... } \\
\text { Ward ..... } \\
\text { Ward ..... } \\
\text { High protein } \\
\text { High protein } \\
\text { High protein } \\
\text { High protein } \\
\text { Low protein } \\
\text { Low protein } \\
\text { Low protein } \\
\text { Low protein } \\
\text { Low protein } \\
\text { Average .... }\end{array}$ & $\begin{array}{l}4 / 9 / 13 \\
4 / 11 / 13 \\
4 / 12 / 13 \\
4 / 13 / 13 \\
4 / 14 / 13 \\
4 / 16 / 13 \\
4 / 17 / 13 \\
4 / 18 / 13 \\
4 / 20 / 13 \\
4 / 23 / 13 \\
4 / 24 / 13 \\
4 / 25 / 13 \\
4 / 26 / 13 \\
4 / 27 / 13 \\
\ldots \ldots \ldots\end{array}$ & $\begin{array}{r}1,740 \\
960 \\
1,020 \\
1,200 \\
1,530 \\
1,440 \\
1,560 \\
1,500 \\
1,920 \\
2,400 \\
1,380 \\
2,400 \\
2,400 \\
1,560 \\
\cdots \ldots\end{array}$ & $\begin{array}{r}13.9 \\
10.5 \\
11.1 \\
9.3 \\
9.5 \\
9.9 \\
12.2 \\
15.5 \\
18.3 \\
12.5 \\
8.4 \\
6.2 \\
4.6 \\
3.4 \\
\ldots . .\end{array}$ & $\begin{array}{c}20 \\
\cdots\end{array}$ & $\begin{array}{l}11 \\
\ldots\end{array}$ & $\begin{array}{c}3.0 \\
\ldots\end{array}$ & $\begin{array}{l}13 \\
\cdots \\
45 \\
33\end{array}$ & $\begin{array}{l}160 \\
139\end{array}$ \\
\hline
\end{tabular}


from our figures, there clearly is no marked connection between the blood-pressure and the degree of retention; indeed, it is doubtful whether there is any connection. A high blood-pressure, therefore, does not necessarily indicate the desirability of introducing low nitrogen diets in nephritis. So far as can be seen at the present time, the only criterion available for regulating the protein content of the food in nephritis is the determination of the amount of retention by blood analysis. Five of the twelve patients (the last five) used in our experiments showed little or no abnormal retention of waste nitrogen, and there is no reason to doubt that these could take care of the protein contained in the usual hospital diet.

So far as the phenolsulphonephthalein test for the kidney efficiency is concerned, it is to be noted that there is no correspondence between the results of this test and the degree of waste nitrogen retention. Since it is possible to increase or reduce the retention by protein feeding one cannot expect any close agreement between it and the phenolsulphonephthalein test. The lack of correspondence is, however, probably not to be explained entirely on the basis of different levels of total protein metabolism.

In Table 13 we give the nitrogen elimination, the non-protein. nitrogen and the phenolsulphonephthalein excretion - all in terms of the average for the first two periods of the experiments.

Table 13.-Average in Cases 1 to 12 of Urinary Nitbogen, Non-Protein Nitrogex and Pheyolsulphonephthalein Excretion*

\begin{tabular}{r|c|c|c}
\hline \hline Case & $\begin{array}{c}\text { Urine } \\
\text { Nitrogen } \\
\text { gm. }\end{array}$ & $\begin{array}{c}\text { Blood } \\
\text { Non-Protein } \\
\text { Nitrogen, mg. }\end{array}$ & $\begin{array}{c}\text { Phenolsulphone- } \\
\text { phthalein Excretion } \\
\text { Per Cent. }\end{array}$ \\
\hline 9 & 8.0 & 28.5 & 27.0 \\
3 & 8.7 & 37.5 & 33.5 \\
2 & 8.9 & 44.0 & 26.2 \\
5 & 9.2 & 54.0 & 19.8 \\
7 & 9.7 & 61.0 & 28.0 \\
1 & 9.8 & 40.0 & 31.6 \\
6 & 10.3 & 50.5 & 30.0 \\
10 & 10.9 & 30.0 & 28.0 \\
8 & 11.0 & 29.0 & 29.0 \\
11 & 11.4 & 31.0 & 29.5 \\
12 & 12.3 & 33.5 & 29.7 \\
4 & 14.3 & 48.5 & 46.6 \\
\hline
\end{tabular}

* Average for first two periods arranged according to urinary nitrogen.

If 60 to 80 per cent. be taken as the normal elimination of $6 \mathrm{mg}$. of phenolsulphonephthalein excretion when injected intramuscularly it is clear that this elimination can be reduced by one-half or more before there need be any abnormal accumulation of waste nitrogen in the blood. 


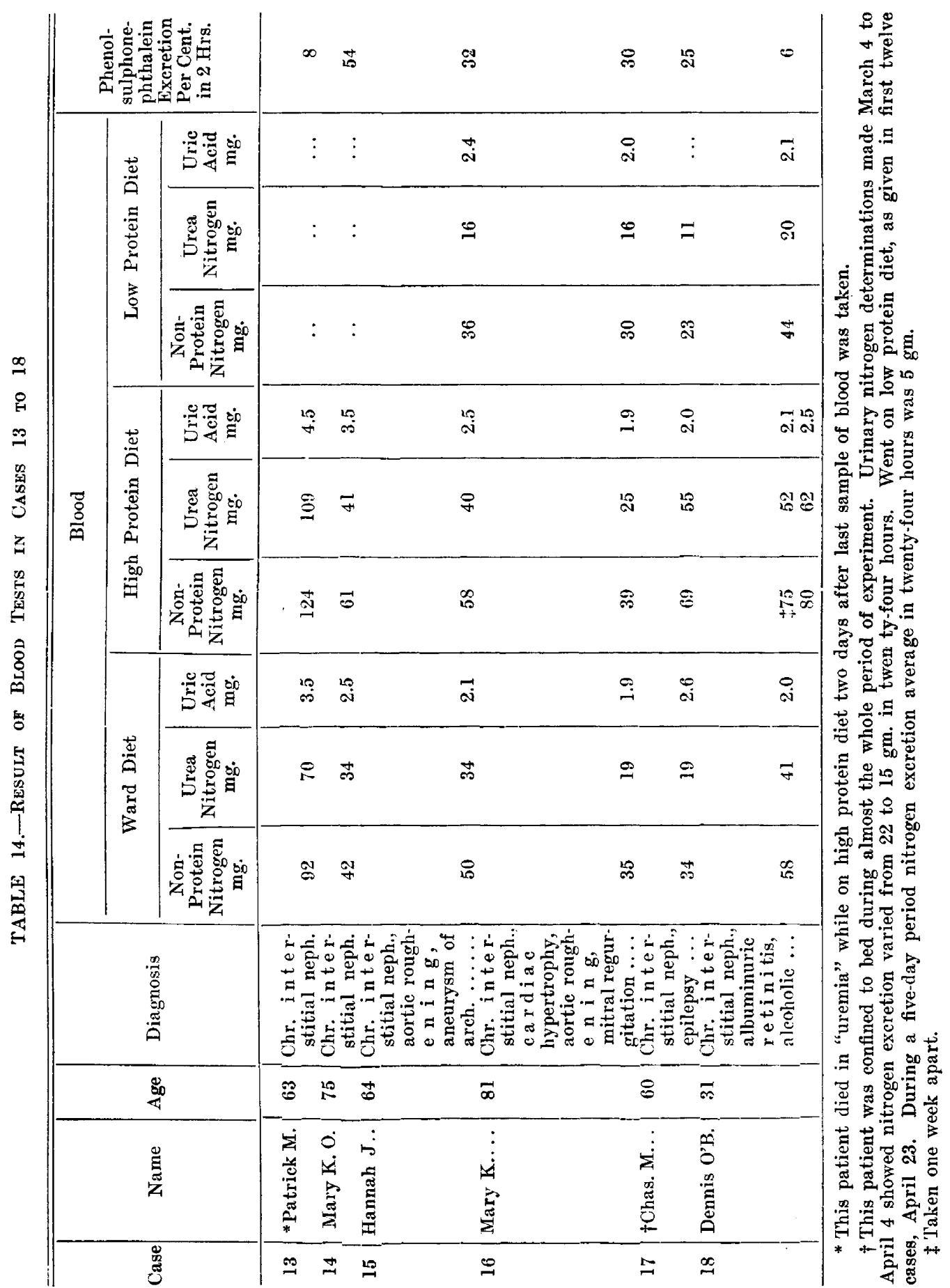


It would seem from these results as though the direct determination of the non-protein nitrogen (and urea) in the blood furnishes a more reliable guide to what might be called the protein tolerance of patients than can be obtained from any "direct" test of kidney efficiency, for of all tests yet devised for this purpose the phenolsulphonephthalein test of Rowntree and Geraghty is admittedly the best.

In addition to the preceding experiments we have made a number of others which are less complete, but from which some noteworthy observations were obtained. These experiments were made before the series shown in Tables 1 to 13 , and while in every case collections of urine were made it was suspected that in a number of instances the whole twenty-four-hour amount had not been saved. We, therefore, have not included our determinations of urinary nitrogen, but have been forced to rely on the dietary records kept by the nurses in charge.

$\mathrm{As}$ in the preceding experiments, eggs and meat were used in liberal amounts during the periods of high protein feeding. In the low protein periods, however, the patients were fed on cereals, bread and butter, vegetables and a little milk. 'The soluble starch employed so extensively in our later experiments was not used, so that in some cases it is to be feared that the calorific intake was too low. 